\title{
Abnormal PL spectrum in InGaN MQW surface emitting cavity
}

\author{
J. T. Chu ${ }^{a}$, Y.-J. Cheng ${ }^{b}$, H. C. Kuo ${ }^{a}$, T. C. Lu ${ }^{a}$, and S. C. Wang ${ }^{a}$ \\ ${ }^{a}$ Department of Photonics \& Institute of Electro-Optical Engineering, National Chiao Tung \\ University, 1001 TA Hsueh Rd., Hsinchu 300, Taiwan; \\ ${ }^{b}$ Research Center for Applied Sciences, Academia Sinica, 128 Sec. 2, Academia Rd., Nankang, \\ Taipei 115, Taiwan.
}

\begin{abstract}
We report the observation of an abnormal photoluminescent (PL) spectrum from a HeCd laser pumped InGaN multiple quantum well (MQW) vertical cavity. The device is fabricated using standard MOCVD deposition on a (0001)-oriented sapphire substrate. The layer structures are: $10 \mathrm{~nm}$ nucleation layer, a $4 \mathrm{um}$ bulk GaN layer, InGaN MQWs, and a final $200 \mathrm{~nm} \mathrm{GaN}$ cap layer. The InGaN MQWs consist of 10 pairs of $5 \mathrm{~nm} \mathrm{GaN}$ barrier and $3 \mathrm{~nm} \operatorname{In}_{0.1} \mathrm{Ga}_{0.9} \mathrm{~N}$ well. The peak emission of the as-grown MQWs sample was $\sim 420 \mathrm{~nm}$. A dielectric distributed Bragg reflectors (DBR) were then coated on the top layer, followed by a laser lift off from sapphire substrate, and subsequently another DBR coated on the bulk GaN bottom surface. The cavity has a quality factor of $\sim 520$ from $400-490 \mathrm{~nm}$. The device was pumped by a focused CW $\mathrm{HeCd}$ laser from the bulk GaN side. When the laser is focused onto the InGaN MQWs, a photoluminescent spectrum centered at the designed MQW wavelength was observed as expected. However, when the focused position was moved toward the bulk GaN region, an additional abnormal PL peak around $460 \mathrm{~nm}$ was observed. This is far outside the designed MQW wavelength.
\end{abstract}

Keywords: InGaN, GaN, MQW, vertical cavity, photoluminescence

\section{INTRODUCTION}

Group III-nitride semiconductor material has attracted great research interests in recent years. This is a promising material system for next generation optoelectronic applications, in particular in the visible to ultra violent region. Its application in solid state lighting has been gaining a lot of progress. It is also an important material for blue-UV laser diodes. The optical properties of III-nitride material are in general still less well investigated compared to other more matured III-V semiconductor in the infrared region. Photoluminescent spectrum is a common method for characterizing the optical properties of a material. It is also known that the radiation properties of an excited material can be modified by a cavity. A well designed cavity can be monolithically integrated to the semiconductor to enhance its radiation efficiency.

Planar semiconductor microcavities have been an attractive device structure for such an application. It has the advantage of circularly symmetric vertical surface emission and small cavity volume to enable strong light-matter interaction and enhance lighting emitting efficiency. The technology for fabricating such a device structure has been well developed for the infrared III-V semiconductor lasers. i.e. vertical cavity surface emitting laser (VCSEL). It has also been demonstrated on III-nitride based devices [1-3]. In this study, we have fabricated InGaN multiple quantum wells in a monolithically integrated microcavity and optically pumped it with $\mathrm{HeCd}$ laser to study the device photoluminescent spectrum. An emission spectrum centered at the designed QW emission wavelength $419 \mathrm{~nm}$ with full width half max bandwidth of $20 \mathrm{~nm}$ was observed when the HeCd laser is focused on InGaN quantum wells. However, when the focus spot was moved away from the QW toward the bulk GaN region, we saw the presence of an unexpected $460 \mathrm{~nm}$ photoluminescent emission peak with also $\sim 20 \mathrm{~nm}$ linewidth. This emission wavelength was not in the designed QW emission. We have studied the variation of these two PL emission peaks versus various vertical pump focus positions and pump power levels.

Vertical-Cavity Surface-Emitting Lasers XII, edited by Chun Lei, James K. Guenter, Proc. of SPIE Vol. 6908, 69080L, (2008) · 0277-786X/08/\$18 - doi: 10.1117/12.762869 


\section{EXPERIMENTS}

The GaInN multiple quantum microcavity was fabricated by standard metal organic chemical vapor deposition (MOCVD) on a (0001)-oriented sapphire substrate followed by dielectric coating runs to form vertical surface microcavity. The process steps are schematically shown in Fig. 1(a). The device layer structure consists of a 30-nm GaN nucleation layer, a 4- $\mu \mathrm{m}$ GaN bulk layer, MQWs consisting of 10 periods of 5-nm GaN barriers and 3-nm $\operatorname{In}_{0.1} \mathrm{Ga}_{0.9} \mathrm{~N}$ wells, and a 200-nm GaN cap layer. A 6-pairs of $\mathrm{SiO}_{2}$ and $\mathrm{TiO}_{2}$ were evaporated on the top of the grown structure to form the top dielectric distributed Bragg reflector (DBR). The reflectivity of the $\mathrm{SiO}_{2} / \mathrm{TiO}_{2} \mathrm{DBR}$ at $419 \mathrm{~nm}$ was $99.5 \%$. Next, an array of circular $\mathrm{SiO}_{2} / \mathrm{TiO}_{2} \mathrm{DBR}$ mesas with diameters of $60 \mu \mathrm{m}$ was formed by standard lithography and chemical wet etching process. To deposit the bottom dielectric DBR, the structure was subjected to a laser lift-off (LLO) process using a $\mathrm{KrF}$ excimer laser to remove $\mathrm{GaN} / \mathrm{InGaN}$ from sapphire substrate. The GaN surface after LLO was further polished to a surface roughness of about $1 \mathrm{~nm}$ for deposition of the bottom dielectric DBR. The bottom DBR consists of 8 pairs of $\mathrm{SiO}_{2}$ and $\mathrm{Ta}_{2} \mathrm{O}_{5}$. The final thickness of the epitaxial structure after LLO and polishing process was about $4 \mu \mathrm{m}$. The $\mathrm{Ta}_{2} \mathrm{O}_{5}$ was used to reduce the absorption of the pumping beam at $325 \mathrm{~nm}$. The reflectivity of the $\mathrm{SiO}_{2} / \mathrm{Ta}_{2} \mathrm{O}_{5}$ DBR at $410 \sim 490 \mathrm{~nm}$ was $97 \%$. The detailed process procedure was described in our previous report [4].

The micro-PL measurement is shown in Fig. 1(b). The InGaN QW microcavities were optically pumped by a CW HeCd laser at $325 \mathrm{~nm}$. The beam size is about $3.5 \mathrm{~mm}$ in diameter after $\mathrm{HeCd}$ laser passes through a beam expander. The pump laser is focused by a $15 \mathrm{X}$ microscope objective. The focused spot size was measured to be $2 \mu \mathrm{m}$ in diameter. The microcavities were pumped from the $\mathrm{SiO}_{2} / \mathrm{Ta}_{2} \mathrm{O}_{5}$ DBR side. The light emission from the VCSEL sample was collected from the same side by an imaging optics into a fiber and delivered to a spectrometer with resolution of $0.05 \mathrm{~nm}$.

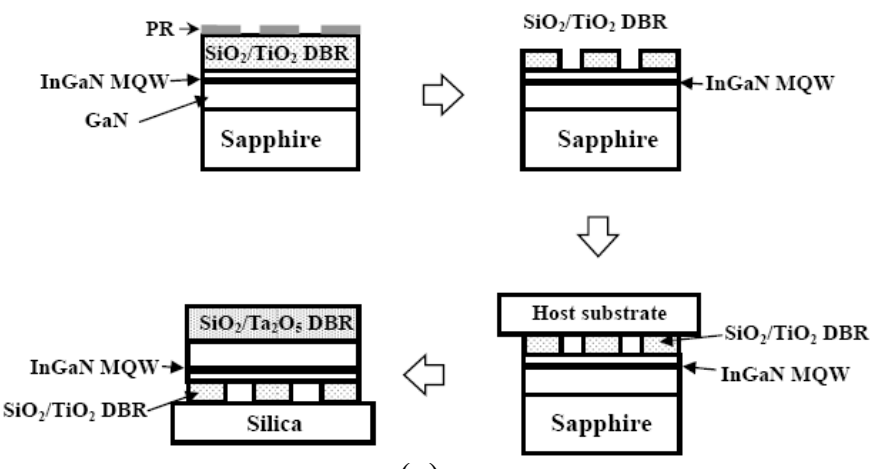

(a)

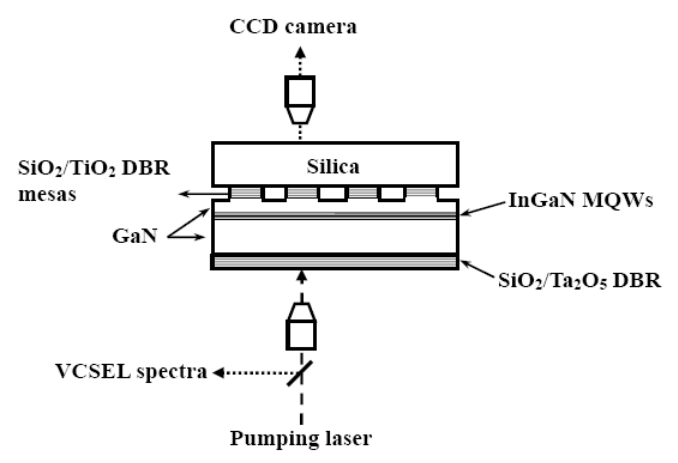

(b)

Fig.1. (a) Fabrication steps of InGaN MQW microcavities. (b) Micro-PL measurement setup. The device was pumped by a focused CW HeCd laser at $325 \mathrm{~nm}$. 


\section{EXPERIMENTS}

We first measured the PL spectrum of an as grown reference sample, which did not have the dielectric DBR cavity. The PL spectrum is shown in Fig. 2. The pump was focused on the InGaN multiple quantum wells. The QW emission was at $419 \mathrm{~nm}$ wavelength. The ripples were due to the Fabry-Perot etalon effect of the $4 \mathrm{um} \mathrm{GaN/InGaN} \mathrm{substrate.} \mathrm{Note} \mathrm{that} \mathrm{there} \mathrm{is} \mathrm{a} \mathrm{small} \mathrm{plateau} \mathrm{from} \mathrm{440nm} \mathrm{to}$ $460 \mathrm{~nm}$ with PL intensity about one third of the $419 \mathrm{~nm}$ peak value. This additional plateau of emission is abnormal. The PL peak at $382 \mathrm{~nm}(3.25 \mathrm{meV})$ is thought to be from the bulk GaN. This peak is close to the reported Zinc Blende GaN band gap energy $3.2 \mathrm{meV}$. There is another much weaker peak at $365 \mathrm{~nm}$, which matches to the often quoted Wurtzite GaN band gap energy $3.4 \mathrm{eV}$. There is another small peak at $391 \mathrm{~nm}$, which might be due to bounded defect states.

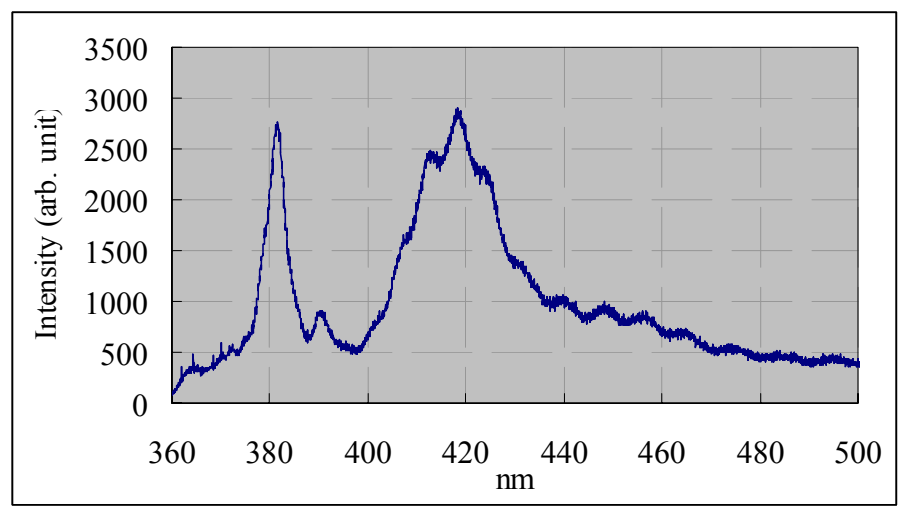

Fig. 2. As grown InGaN multiple quantum well PL. The ripples were due to the Fabry-Perot etalon effect of the 4um $\mathrm{GaN} / \mathrm{InGaN}$ substrate.

We then measured the PL of an InGaN MQW microcavity. The pump was again focused on the InGaN multiple quantum wells. The Spectrum is shown in Fig. 3. The expected strong cavity resonant modes were observed. However, the $382 \mathrm{~nm}$ peak was not as clearly present and there was an additional broader photoluminescent peak at $365 \mathrm{~nm}$ with ripples on its right wing.

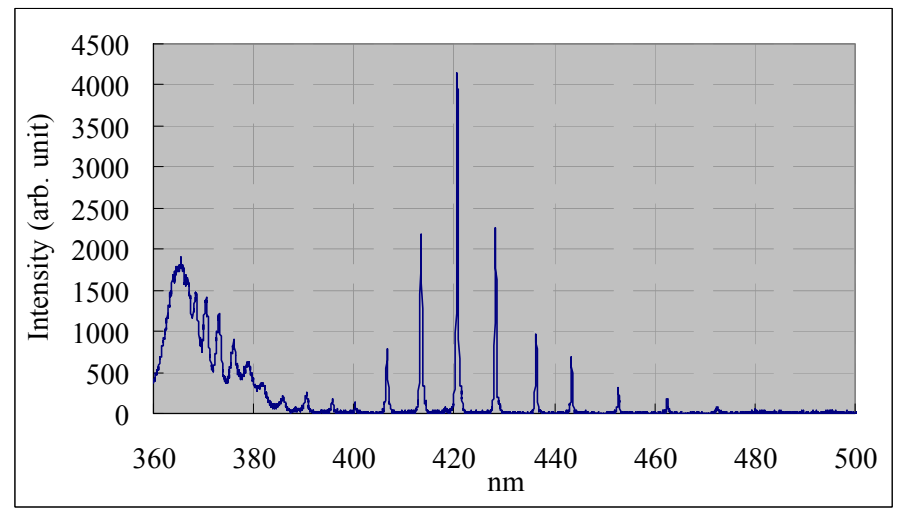

Fig. 3 The PL spectrum of an InGaN multiple quantum well microcavity. The pump was focused on the multiple quantum well.

The focus of the pump laser was gradually moved away from the MQW toward the bulk GaN direction. The typical spectra were shown from Fig. 4 (a) to (f) at various positions as labeled in the plots. The $419 \mathrm{~nm}$ peak value decreased fairly fast when the focus position was moved away from MQWs, while the $460 \mathrm{~nm}$ peak increased but not as fast. The increase of the abnormal $460 \mathrm{~nm}$ peak is rather unusual. The peak monotonically increased until the pump focus spot at $135 \mathrm{um}$ away from the quantum well position, which was far outside the microcavity. 


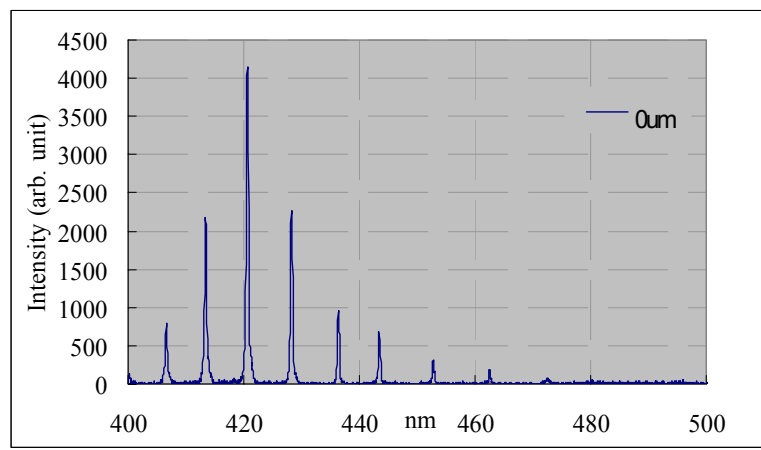

(a)

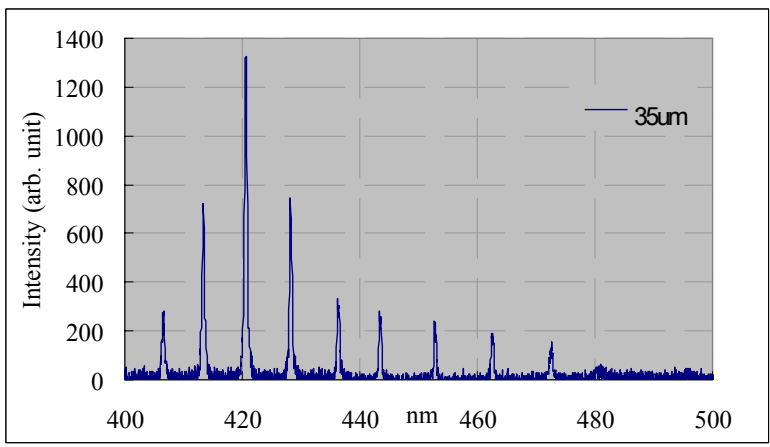

(c)

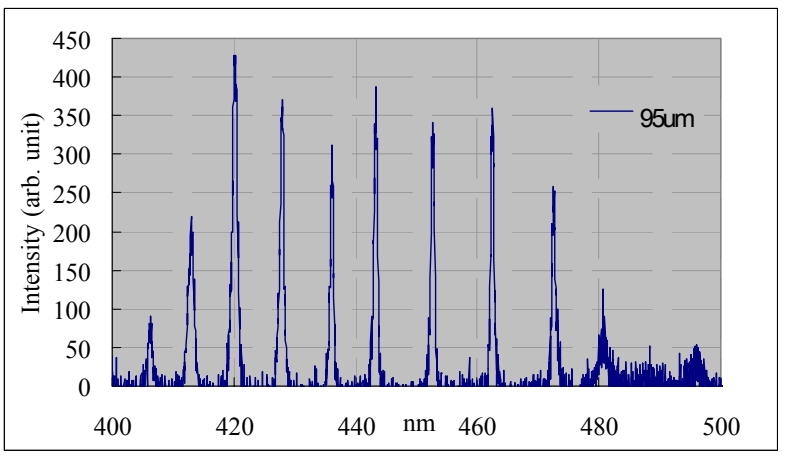

(e)

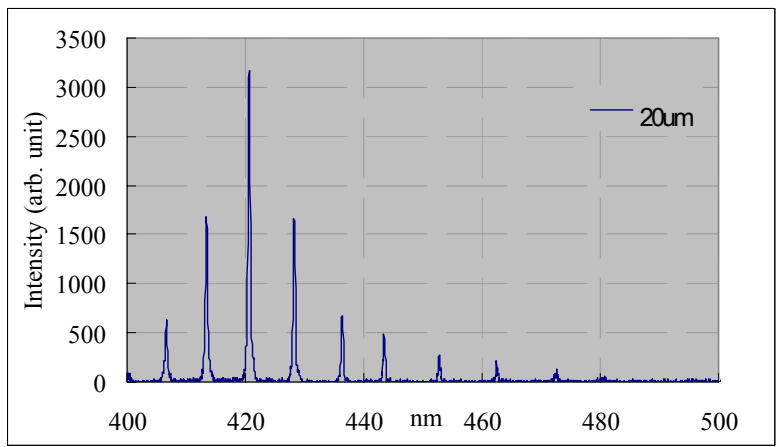

(b)

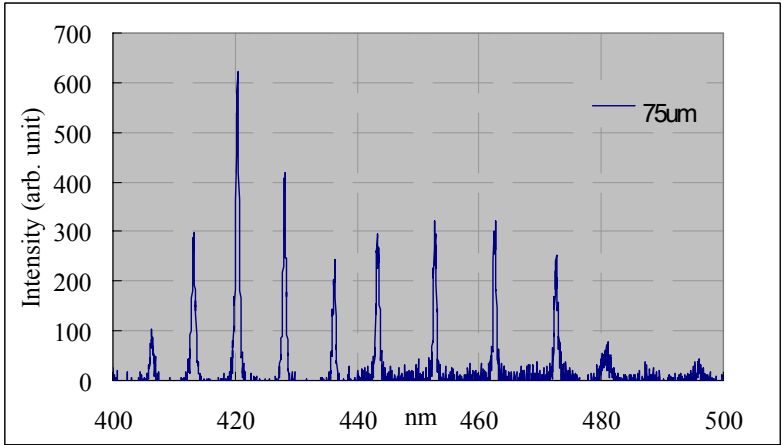

(d)

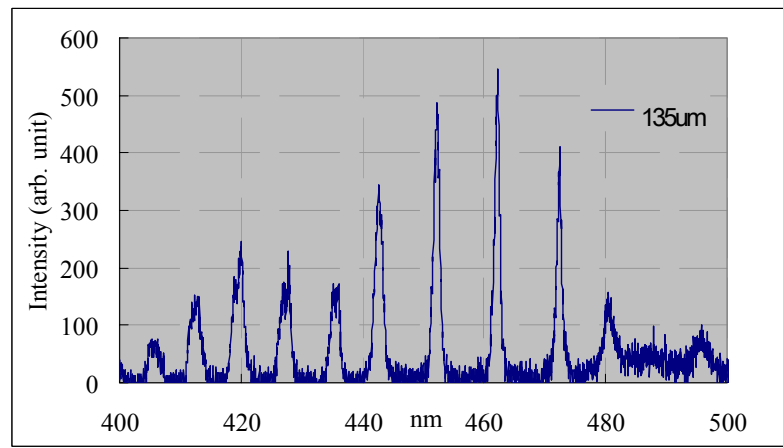

(f)

Fig. 4. (a)-(f) are the PL spetra at various pump focus positions. The labels are the pump focus position measured from the quantum well toward the bulk $\mathrm{GaN}$ region. The quantum well $420 \mathrm{~nm}$ peak decreases when pump focus is moved away from quantum well, while the abnormal $460 \mathrm{~nm}$ peak increases.

\section{CONCLUSION}

We have fabricated InGaN multiple quantum well microcavities to study the device photoluminescent property. The device was pumped by a focused $\mathrm{HeCd}$ laser at $325 \mathrm{~nm}$. The cavity resonant emission at the quantum well designed wavelength at $419 \mathrm{~nm}$ was observed as expected. However, the excited cavity axial modes can extend to well outside the quantum well emission bandwidth. More over, when the pump focus was moved away from the quantum well region, i.e. a divergent pump beam, an unusual emission peak outside the quantum well wavelength at $460 \mathrm{~nm}$ was observed. This peak increases as the pump focus is moved away from quantum well, while the quantum well emission decreases. This unusual behavior might be related to certain nonlinear property of the $\mathrm{GaN}$ material and requires further study. 


\section{REFERENCES}

1. J. M. Redwing, D. A. S. Loeber, N. G. Anderson, M. A. Tischler and J. S. Flynn: Appl. Phys. Lett. 691 (1996).

2. I. L. Krestnikov, W. V. Lundin, A. V. Semenov, A. S. Usikov, A. F. Tsatsul'nikov, N. N. Ledentsov, A. Hoffmann and D. Bimberg: Appl. Phys. Lett. 751192 (1999).

3. T. Someya, R. Werner, A. Forchel, M. Catalano, R. Cingolani and Y. Arakawa: Science 2851905 (1999).

4. Y. K. Song, H. Zhou, M. Diagne, A. V. Nurmikko, R. P. Schneider, Jr., C. P. Cuo, M. R. Krames, R. S. Kern, C. Carter-Coman and F. A. Kish: Appl. Phys. Lett. 761662 (2000).

5. T. Tawara, H. Gotoh, T. Akasaka, N. Kobayashi and T. Saitoh: Appl. Phys. Lett. 83830 (2003).

6. Jung-Tang Chu, Tien-Chang Lu, Min You, Bor-Jye Su, Chih-Chiang Kao, Hao-Chung Kuo, and Shing-Chung Wang,"Emission characteristics of optically pumped GaN-based vertical-cavity surface-emitting lasers," Appl. Phys. Lett. 89, 121112 (2006). 